\title{
New Measures in Controlling Quality of VLT VISIR
}

\author{
Danuta Dobrzycka $^{a}$, Leonardo Vanzi ${ }^{b c}$, Lars Lundin $^{a}$, \\ Hans Ulrich Kaeuf ${ }^{a}$ and Ralf Siebenmorgen ${ }^{a}$ \\ ${ }^{a}$ ESO, Karl Schwarzschild Str. 2, 85748 Garching, Germany \\ ${ }^{b}$ ESO, Alonso de Cordova 3107, Santiago 19, Chile \\ ${ }^{c}$ Pontificia Universidad Catolica de Chile, Av. Vicuna Mackenna 4860, Santiago, Chile
}

\begin{abstract}
The ESO's VISIR instrument at Paranal is dedicated to observations in two mid-infrared (MIR) atmospheric windows: N-band (8-13 micron) and Q-band (16.5-24.5 micron). It is equipped with two DRS (formerly Boeing) $256 \times 256$ BIB detectors operating at temperatures of about $5 \mathrm{~K}$. As in case of other Paranal instruments VISIR data are regularly transferred to ESO Garching within the standard data flow operation. There, they are classified and pipeline-processed. The products of VISIR technical data are analyzed in order to trend instrument performance, while calibrations and science data are checked for quality and later distributed to the users. Over the three years of VISIR operations we have been constantly gaining more experience in methods of assessing health of the instrument. In particular, we found that dark frames are particularly useful for monitoring the VISIR detectors. We also discuss performance of the "OCLI" silicate filters recently mounted in the instrument.
\end{abstract}

Keywords: quality control, VISIR, trend analysis, data reduction pipelines, instrument performance

\section{INTRODUCTION}

The VLT Spectrometer and Imager for the Mid-Infrared (VISIR) is an instrument dedicated to observations through the two mid-infrared (MIR) atmospheric windows: $\mathrm{N}$ band $(8-13 \mu \mathrm{m})$ and $\mathrm{Q}$ band $(16.5-24.5 \mu \mathrm{m})$. It is installed at ESO Paranal Observatory at the Cassegrain focus of the 3rd VLT Unit Telescope - Melipal. This cryogenic instrument combines diffraction limited high sensitivity imaging capabilities over a field of view of up to $51^{\prime \prime}$ and long slit grating spectroscopy capabilities with a range of spectral resolutions between 150 and 30000 .

VISIR consists of two sub-instruments: imager and spectrometer. They have independent light paths, optics and detectors. The cryogenic optical bench is enclosed in a vacuum vessel and cooled to $33 \mathrm{~K}$ for most of the structure and optics. Both imager and spectrometer are equipped with the DRS (formerly Boeing), $256 \times 256$ BIB detectors. Their quantum efficiency is greater than $50 \%$, reaching $65 \%$ or more at $12 \mu \mathrm{m}$. The detectors were originally cooled down to $\sim 7 \mathrm{~K}$. It was decreased to $\sim 5 \mathrm{~K}$ in December 2005. Both DRS detectors have a fair fraction of bad pixels and display artifacts like, for example, striping, appearances of ghosts, fringes, etc. These artifacts are smaller in spectroscopy due to the lower flux level but are clearly visible on objects brighter than $\sim 2 \%$ of the background or when the hot pixels are read. Also in case of integrating on bright objects the detectors show memory effect - increased number of counts lingering for extended time. ${ }^{1,2}$

The imager consists of two parts: collimator, providing an $18 \mathrm{~mm}$ diameter cold stop pupil in parallel light and a wheel with three objectives. The $0.075^{\prime \prime}$ (small field, SF) and $0.127^{\prime \prime}$ (intermediate field, IF) pixel scales are offered. The filter wheel is located just behind the cold stop pupil mask. Currently 23 filters are available and additional and/or replacement filters are considered.

Spectrometer offers slit spectroscopy at three spectral resolutions with a pixel scale of $0.127^{\prime \prime}$. The instrument contains two arms: one with low order gratings for the low and medium spectral resolution and the other with large echelle gratings providing high spectral resolution. Both subsystems image the spectrum onto the same detector. Selection between the two arms is done by two pairs of folding flat mirrors. The spectrometer slit wheel is also equipped with a very wide slit $\left(15.3^{\prime \prime}\right)$, which enables imaging with the spectrometer detector. This capability is used for object acquisition and centering on the detector.

D.D.: E-mail: ddobrzyc@eso.org, Telephone: +49 8932006587 
From the beginning, VISIR operations were designed to follow the scheme common to all VLT instruments. The scheme includes not only instrument maintenance at Paranal but also the data $\mathrm{flow}^{3}{ }^{4}{ }^{4}$

In this paper we present application of particular VISIR calibrations - dark frames - for monitoring of the health of the VISIR's two MIR detectors. We also introduce and describe performance of the "OCLI" silicate filters that were mounted in VISIR during the technical intervention last year.

\section{DATA HANDLING AND QUALITY CONTROL}

From the moment VISIR was offered to the community in April 2005 its operation followed the scheme common to all the VLT instruments. The process of handling, processing and analysis of data has been described in details in e.g. ${ }^{45}$

The observations are obtained either in visitor or service mode. The visitor mode data are packed on-site and collected by the visiting astronomer, while all the calibration frames and service mode data are transferred to ESO in Garching for further processing and inspection by the Data Flow Operation Quality Control Group. Here, the data are classified and reduced using pipeline. The quality control (QC) parameters calculated by the recipes are extracted from the headers of the pipeline products. They are automatically stored in the QC1 database, which is publicly available at http://archive.eso.org/bin/qc1_cgi

Subsequent quality control process includes several activities: assessing the quality of the raw data (also done at Paranal), checking quality of data products created by pipelines, processing as well as distribution of the service mode data to the principal investigators, and - one of the most important goals - monitoring performance of the instrument through temporal behavior of the QC parameters. ${ }^{6}$

Monitoring of health of the different components of the instrument is mainly done by designing particular quality parameters that reflect performance of these components and further, by following their variations with time. They are plotted as Health Check plots and can be found at:

$$
\text { http://www. eso.org/observing/dfo/quality/ALL/daily_qc1.html }
$$

In the current VLT operation scheme the FITS files arrive from Paranal to Garching with a delay of few days. That creates a time gap between the QC parameters derived in Garching and the most recent ones. To assure the up-to-date coverage we include the values from the on-line pipeline processing at the Paranal from last night. Within time the data points from Paranal are replaced by the ones extracted in Garching. The Health Check plots are refreshed frequently, whenever new data points are detected (checks are done once per hour). In their latest version the Health Check plots also contain additional information, like historical plots of selected QC parameters or tutorial pages that explain in detail how the parameters are calculated, analyzed, etc.

\section{MONITORING VISIR DETECTORS}

Over the three years of VISIR operations we have been constantly gaining more experience in methods of assessing health of the instrument and detectors in particular. $\mathrm{In}^{4}$ we reported the list of VISIR calibrations and QC parameters that were being monitored. Since then, we have introduced new type of VISIR calibration data - dark frames. They are utilized as technical data and are not meant to be used in calibrating science observations. The darks were first included in the standard set of day time calibrations on August 24, 2007. Originally, the template consisted of 2 raw dark frames taken with following instrumental setup:

- imaging detector: INS.PFOV=0.127", DET.DIT=0.02, DET.NDIT=1000,

- spectroscopic detector: INS.PFOV=0.127", DET.DIT=0.02, DET.NDIT $=1000$, INS.RESOL $=\mathrm{MR}$ and HRS, INS.FILT2.NAME=N_SW and ARIII, INS.GRAT1.WLEN=7.8 and 8.9 respectively. 
VISIR: (last 180 days)

QC data range: 2007-12-07 ... 2008-05-29; last OPSLOG data: 2008-06-02

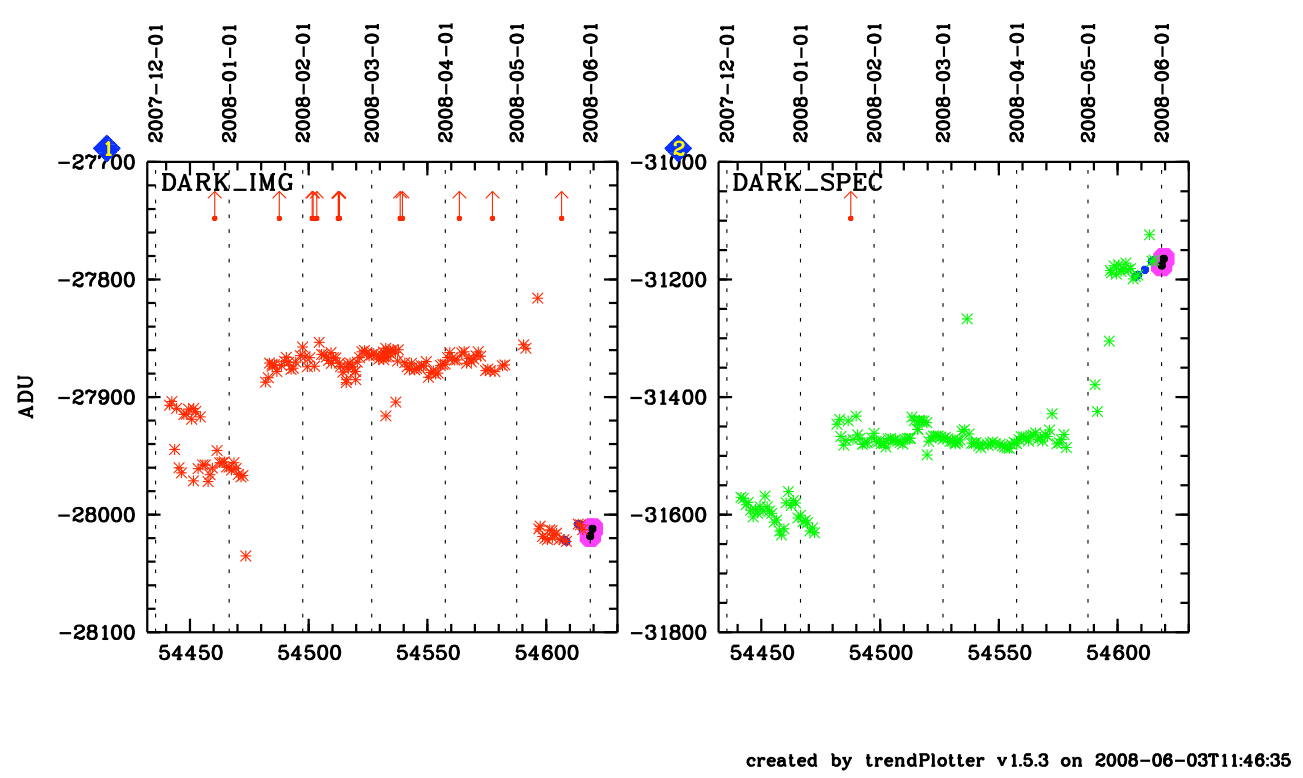

Figure 1. Example of a VISIR's Health Check plot - mean dark level. The left panel shows the values calculated from the dark frames of the imaging detector, while the right panel values calculated from the darks of the spectroscopic detector taken in high resolution slit (HRS) mode. The most recent points (filled circles) correspond to values from Paranal on-line pipeline runs and stars come from regular processing by QC Garching. The arrows point to outliers. The shaded large circles mark the latest data points available on the date the plot was created.

The keyword INS.PFOV corresponds to pixel scale, DET.DIT is the detector integrations time, DET.NDIT is the number of sub-integrations and INS.RESOL points to the medium resolution (MR) or high resolution slit (HRS). The MR and the HRS setups were chosen to include two spectrometer arms. For the MR, filter (INS.FILT2.NAME) N_SW and grating (INS.GART1.WLEN) centering at $7.8 \mu \mathrm{m}$ were selected, while for HRS, filter ARIII and grating $8.9 \mu \mathrm{m}$.

Usefulness of darks to monitor performance of the MIR detectors has not been yet clearly demonstrated. This is because the intrinsic detector signal can be embedded in the background due to photons emitted by the telescope and the atmosphere.

Two standard parameters determined from the dark frames are monitored:

- mean dark level (DARK): it is calculated in the central part of the combined dark frame,

- read-out-noise $(\mathrm{RON})$ : it is a median of the standard deviations measured from hundred $4 \times 4$ windows on the difference frame, multiplied by sqrt(NDIT/2). Both differences of the 2 nd -1 st and 3rd-2nd raw frames are used to calculate two read-out-noise values. The values are determined the same way for the imaging and spectroscopic detector.

Figure 1 is an example of the standard VISIR Health Check plot showing behavior of the mean dark level of the imaging (DARK_IMG) and spectroscopic (DARK_SPEC) detectors during the last 180 days. Also the historical plots (showing data points older than 6 months) are available from the standard QC Health Check web site. The panel DARK_SPEC displays only the dark level for the HRS mode because the dark level for MR mode has quite similar values. The negative values of the dark counts come from the fact that the offset correction (32768 value added for each pixel) has not been applied. For completeness the plot contains data points coming 
VISIR: RON values (last 180 days)

QC data range: 2007-12-07 ... 2008-05-29; last OPSLOG data: 2008-06-02
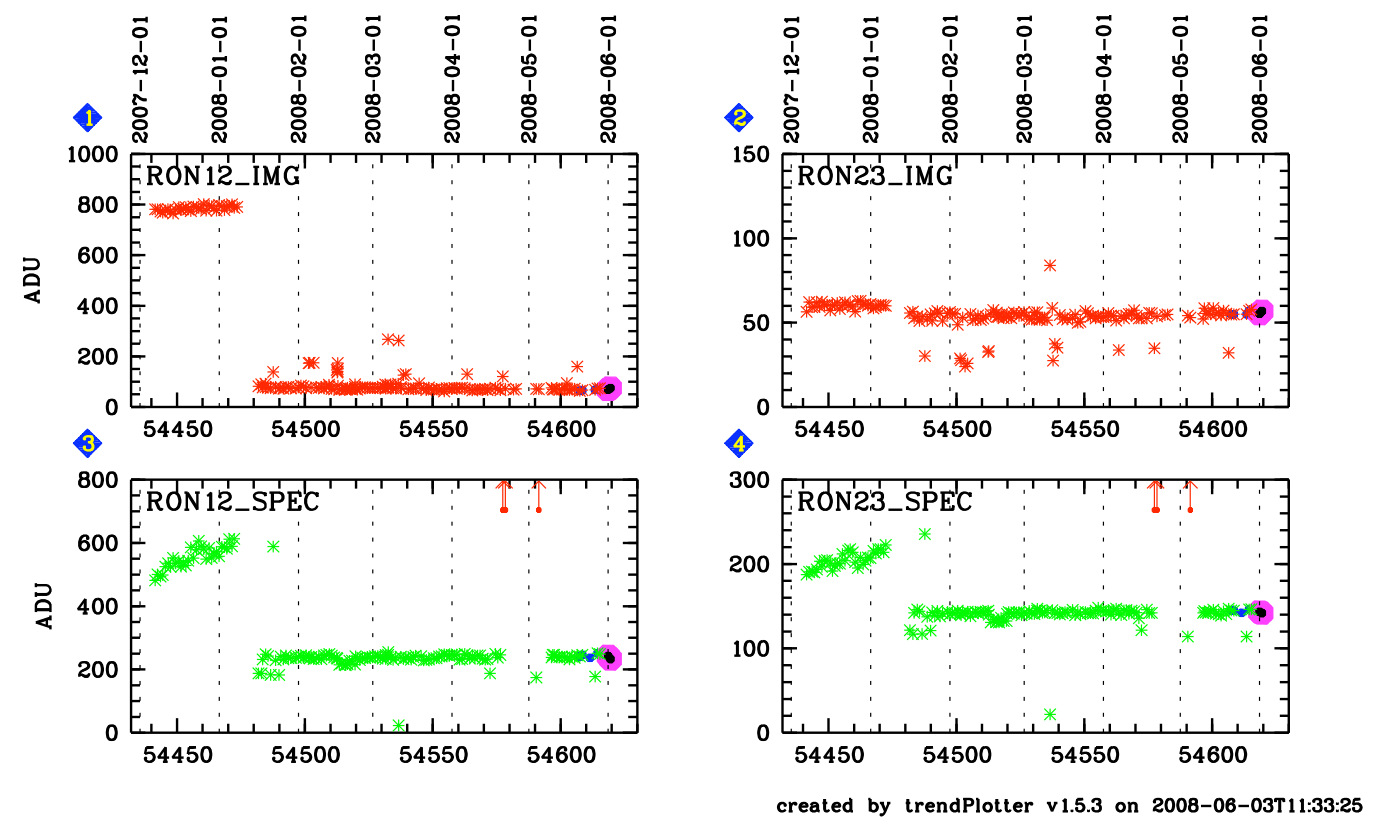

Figure 2. Combination of two Health Check plots of VISIR monitoring read-out-noise values (RON) determined from the darks of the imaging (IMG) and spectroscopic (SPEC) detectors. The left panels show read-out-noise values calculated from the first and second raw frames (RON12) and the right panels values calculated from the second and third raw frames (RON23). The most recent points (filled circles) correspond to values from Paranal on-line pipeline runs and stars come from regular processing by QC Garching. The shaded large circles mark the latest data points available on the date the plot was created.

from regular processing in Garching (star symbols) and up to date values generated by the on-line pipeline runs in Paranal (dots) ftp-ed to Garching once an hour in form of the OPSLOG files. The header of the plot describes the date range for the data points used.

There are two jumps visible in the dark level. The first occurred around January 18, 2008 and corresponds to power outage at Paranal. The second occurred on May 6, 2008, when a "high risk" earthquake was felt on the mountain. The variations clearly showed us sensitivity of the detectors to both events. In each case the change was analyzed and assessed. It was decided that the relative amplitude of the variations was small and no further steps were necessary. The arrows point to occasional outliers. They are also investigated and followed in time.

Originally, the VISIR dark template consisted of two raw dark frames. However, we've noticed that the first dark raw frame of the stack is consistently contaminated by a transient feature, due to detectors' memory effect, which artificially increases the background level. The feature seems to disappear already in the second frame so started from November 7, 2007 we've introduced the third frame into the template. From then on we have been monitoring the read-out-noise determined from both: differences of the 2nd-1st (RON12) and 3rd-2nd (RON23) raw frames. Figure 2 shows the values of those parameters as a function of time over the last 180 days for the imaging and spectroscopic detectors. Note that the RON12 values are much higher than RON23, but they consistently show similar variability. Again, the RON of the spectroscopic detector refers only to the HRS mode. The RON values of the MR mode are about 40 counts higher, but have the same temporal behavior.

Figure 2 clearly shows that the read-out-noise dropped significantly as a result of the January 18, 2008 power outage. This change prompted us to assess the new state of the detectors. Since the read-out noise reduced and stabilized it was concluded that the state of detectors improved. The RON values showed only minor sensitivity 
to the May 6, 2008 earthquake.

Overall, we've found the QC parameters determined from the dark frames a useful tool in following performance of the VISIR detectors. Their monitoring is planned to be continued with possible development of additional calibrations and QC parameters.

\section{VISIR SILICATE FILTERS}

Between April, 24 and May 8, 2007 VISIR underwent a technical intervention. In its course a set of silicate filters, known to the community as the "OCLI filter set", was installed. Silicate dust is a common component of many astronomical objects, from the comets to the active galactic nuclei. It can be identified by a strong feature observed in the atmospheric $10 \mu \mathrm{m}$. Depending on circumstances the feature is seen in absorption or in emission. As a mid-infrared instrument VISIR is well suited for this kind of observations. However lack of the silicate filters made them impossible, seriously affecting VISIR's scientific productivity.

Initially, the new filters were tested off-sky using the extended calibration source. It was found that the J7.9 filter creates a noticeable filter ghost $(\approx 5 \%)$ and the J8.9 filter introduces a significant offset causing the field mask to produce a shadow. The shadow affects the detector image, making the use of the filter questionable.

Further, the new filters were used in the on-sky observations. The optimal observing parameters (combination of the detector integration time and detector capacity) were determined and a number of standard star observations were acquired. The data were pipeline processed to calculate sensitivities. We reported the initial results in. ${ }^{7}$ However, since that time the silicate filters have been included in the standard operations, and many more imaging standard star observations were taken. All sensitivities determined from those observations were ingested in the VISIR QC1 database, ${ }^{5}$ where they are publicly available for analysis.

Table 1 contains characteristics of the new filters and up to date estimates of the sensitivities. Only observations taken in weather conditions flagged as "good" were taken into account. The results are separated by the available pixel scales: $0.075^{\prime \prime}$ (SmallField) and 0.127" (Interm.Field). For the B8.7 filter we still miss the zero point fluxes and therefore we cannot calculate sensitivities.

Table 1. The characteristics of the new filters and up-to-date measured sensitivities. Only observations acquired in the weather conditions flagged "Good" are considered.Sensitivities are expressed in mJy for a $10 \sigma$ detection in 1 hr exposure time and are given for the two VISIR magnifications (0.075 - SmallField, 0.127 - Interm.Field). In each case the best measured and the median values are given. The wavelength range of the filters is given in $\mu \mathrm{m}$.

\begin{tabular}{|l|l|l|l|l|l|l|}
\hline Filter & Cut on & Cut off & Sensitivity & Sensitivity & Sensitivity & Sensitivity \\
\hline Name & $50 \%$ & $50 \%$ & SmallField & SmallField & Interm.Field & Intermd.Field \\
\hline & & & Best & Median & Best & Median \\
\hline J7.9 & 7.483 & 8.035 & 11.1 & 18.0 & 21.0 & 38.8 \\
\hline J8.9 & 8.338 & 9.068 & 2.3 & 3.2 & 4.2 & 5.7 \\
\hline J9.8 & 9.123 & 10.059 & 5.4 & 6.8 & 9.0 & 10.9 \\
\hline J12.2 & 11.700 & 12.216 & 11.0 & 11.9 & 18.6 & 18.6 \\
\hline B8.7 & 8.436 & 9.410 & no data & no data & no data & no data \\
\hline B9.7 & 9.402 & 10.242 & 6.7 & 10.1 & 16.4 & 25.8 \\
\hline B10.7 & 9.970 & 11.338 & 4.0 & 5.0 & 7.6 & 11.8 \\
\hline B11.7 & 11.098 & 11.950 & 3.9 & 4.8 & 5.9 & 7.9 \\
\hline B12.4 & 11.971 & 12.961 & 7.5 & 10.2 & 10.2 & 12.9 \\
\hline
\end{tabular}

The new scientific capability of VISIR was successfully verified immediately after VISIR returned to sky observations following the April-May 2007 instrument intervention. ${ }^{78}$ used the VISIR silicate filters together with other imaging filters to map - for the first time - the atmospheric temperatures of Neptune at different 
altitude levels. It was found that the south pole of Neptune, at the level of tropopause, appears to be warmer than the rest of the planet by 6 to $8 \mathrm{~K}$. Also, the authors found the evidence for a Neptune stratospheric hot spot located at $65-70^{\circ} \mathrm{S}$, corotating with the planet.

\section{SUMMARY}

It has been three years since VISIR - the mid-infrared imager and spectrometer - was introduced at Paranal and offered to the community. From the very beginning of its operations it has been integrated in the standard model of the VLT data flow. The data are transferred to Garching, there they are pipeline processed, checked for quality and, in case of the service mode programs, distributed to the users by the QC group in Garching. One of the major goal of quality control is monitoring health of the components of the instruments. It is mostly done by designing specific calibration or technical observations and following behavior of the QC parameters determined from these data. Since August 2007 we have introduced new VISIR calibrations - dark frames. Two monitored QC parameters: read-out-mode and dark level proved to be useful tools in following performance of the VISIR's MIR detectors. As we presented here, so far they have allowed us to assess state of the detectors after power outage and "high risk" earthquake at Paranal this year.

During a technical intervention last year a set of the "OCLI" silicate filters was mounted on VISIR. Those filters significantly improved VISIR's scientific productivity. We presented their up-to-date measured sensitivities.

\section{REFERENCES}

[1] European Southern Observatory, VISIR User Manual, VLT-MAN-ESO-14300-3514, Version 81 (Feb. 2007). http://www.eso.org/instruments/visir/doc/.

[2] Lagage, P. O., Pel, J. W., Authier, M., Belorgey, J., Claret, A., Doucet, C., Dubreuil, D., Durand, G., Elswijk, E., Girardot, P., Käufl, H. U., Kroes, G., Lortholary, M., Lussignol, Y., Marchesi, M., Pantin, E., Peletier, R., Pirard, J.-F., Pragt, J., Rio, Y., Schoenmaker, T., Siebenmorgen, R., Silber, A., Smette, A., Sterzik, M., and Veyssiere, C., "Successful Commissioning of VISIR: The Mid-Infrared VLT Instrument," The Messenger 117, 12-16 (Sept. 2004).

[3] Quinn, P. J., Albrecht, M. A., Ballester, P., Banse, K., Chavan, A. M., Grosbol, P., Peron, M., and Silva, D. R., "VLT Data Flow System: from concepts to operations," in [Observatory Operations to Optimize Scientific Return], Quinn, P. J., ed., Proc. SPIE 3349, 2-9 (July 1998).

[4] Dobrzycka, D., Smette, A., Sterzik, M., Lundin, L., Jung, Y., and Siebenmorgen, R., "VLT VISIR - controlling data quality and instrument performance," in [Observatory Operations: Strategies, Processes, and Systems], Silva, D. R. and Doxsey, R. E., eds., Proc. SPIE 6270, 26-32 (July 2006).

[5] Dobrzycka, D. and Vanzi, L., "Calibrating mid-infrared standard stars," in [The 2007 ESO Instrument Calibration Workshop], Kaufer, A. and Kerber, F., eds., Proc. The 2007 ESO Instrument Calibration Workshop 28 (Jan. 2008).

[6] Hanuschik, R. and Silva, D., "VLT quality control and trending services," The Messenger 108, 4-9 (June 2002).

[7] Kaufl, H.-U., Nurnberger, D., Beksai, P., Dobrzycka, D., Jimenez, J., Leiva, A., Lundin, L., Marchesi, M., Mardones, P., Mehrgan, L., Pirard, J.-F., Rojas, C., Salazar, D., Siebenmorgen, R., Silber, A., van den Ancker, M., Weilenmann, U., Durand, G., Pantin, E., and Moerchen, M., "Peering into the Dust: News from VISIR," The Messenger 130, 8-11 (2007).

[8] Encrenaz, T., Orton, G. S., Leyrat, C., Puetter, R. C., Friedson, A. J., and Pantin, E., "First thermal IR images of Neptune: Evidence for southern polar heating and methane escape," The Messenger 130, 23-26 (2007). 